\title{
Menus for Managing Patients With Cutaneous T-Cell Lymphoma
}

\author{
Brian Poligone, MD, PhD, ${ }^{*}$ and Peter Heald, $\mathrm{MD}^{\dagger}$
}

\begin{abstract}
In the management of patients with cutaneous T-cell lymphoma (CTCL), there are numerous distinct therapy options. Each of these therapies is discussed in terms of when to use it, what factors limit the success of the treatment, and what to expect. A menu is defined as a list of items from which to choose. The treatments for CTCL are presented in various menus where they are options for a particular goal in a particular setting of CTCL. The best recognized clinical scenarios of CTCL are those recognized by the staging system: limited patch plaque (T1), disseminated patch plaque (T2), erythroderma (T4), and tumor (T3). Each phase of the disease will have the menu of therapy options presented for a given goal of management.
\end{abstract}

Semin Cutan Med Surg 31:25-32 @ 2012 Published by Elsevier Inc.

KEYWORDS cutaneous T-cell lymphoma, mycosis fungoides, Sezary syndrome, phototherapy, topical chemotherapy, radiation therapy, bexarotene, vorinostat, romidepsin, pralatrexate

$\mathrm{T}_{\mathrm{i}}^{\mathrm{h}}$ complex nature of the immunology of the skin is represented in the malignancy of one of the key participants-cutaneous T-cells-in cutaneous immune responses. The identification of malignant $\mathrm{T}$-cells bearing an identifiable skin-homing receptor led to the realization that the varied diseases incorporated in the term cutaneous T-cell lymphoma (CTCL) are clonal expansions of cutaneous Tcells. ${ }^{1}$ By far, the most common CTCL is mycosis fungoides (MF), and most of the studies discussed here are based on studies of MF patients. The presentations of the management menus are to reflect the approach to the patient with CTCL. The first step is to identify the subset of CTCL, the extent of disease, the measurable parameters, the goal of therapy, and then the choice of therapy from a menu. Of the CTCL sub-

* Department of Dermatology, University of Rochester School of Medicine Rochester, NY.

$\uparrow$ Department of Dermatology, Yale University School of Medicine, New Haven Milford, CT

Conflict of Interest Disclosures: The authors have completed and submitted the ICMJE Form for Disclosure of Potential Conflicts of Interest. Dr Heald's institution has received grants from Merck, Therako and Eisai for various aspects of this manuscript. Dr. Heald reported receiving consulting fees or honoraria from Merck, Eisia, Abbott, Centocor and Amgen; has received fees for participating in review activities from Astellas; has received payment for serving on speakers bureaus from Amgen, Abbott and Centocor; and has received payment for developing educational presentations from Amgen. Dr. Poligone has nothing to disclose.

Address reprint requests to Peter Heald, MD, Department of Dermatology, Yale University School of Medicine 212 Sawyer Hill Road, New Milford, CT 06776.E-mail: peter.heald@yale.edu sets, the TNM staging system only applies to straightforward $\mathrm{MF}$, not any of the histologic or clinical variants. However, the severity of the disease, whether MF or a variant, is captured by the T status, with limited T1 disease being the best and universal skin involvement $\mathrm{T} 4$ and transformed disease T3 having a guarded prognosis. ${ }^{2}$

The goals of therapy are defined by the parameters that measure them. Goals of treatment need not be fixed and can change at any time because of the myriad of influences that affect goal selection. Understanding the differences in achieving and measuring different goals is critical to successful management of a patient with CTCL. To illustrate the difference in goal-driven therapy, consider 2 approaches to a patient with disseminated patches of MF. One may be applying topical steroids as needed at home, whereas the other may travel to a facility that specializes in electron beam therapy to undergo a treatment that induces hair loss, requires 9 weeks of devoted time, and reliably induces a remission. The difference between the 2 is the difference in therapy goals: palliation in the first case and remission in the second.

Palliation and remission are 2 distinctly different goalsone is measured by the quality of life and the other by tumor burden. The goal of therapy (palliative or remittive) is the first decision to be made with a patient. There are numerous studies that document cures in CTCL. Most long-term therapy trials define cure as 8 years disease-free and off all therapies. ${ }^{2}$ The first step to cure is a complete remission, meaning that the tumor burden assessment is at zero. When tumor burden is reduced partially, it often is reflected in patient- 
reported outcomes (PROs) as a palliative response. There have been no studies that document a positive impact on a patient from partial tumor burden reduction outside of the effect on improving quality of life. Hence, all partial responses are evaluated by their palliative impact. For some therapies, side effects may complicate the quality of life. Administration and toxicity issues need to be heavily weighed in a palliative plan of therapy. Thus, the 2 goals of therapy have their own primary parameter. For remission, it is tumor burden, and typically that is some assessment of body surface area. For palliation, it is PROs, typically quality of life assessments. Parameters of palliation and remission have been validated in clinical trials, and these can be used in the management of patients outside of clinical trials.

The selection of a goal involves several variables that involve patients, their family, and where they live. The latter is important in terms of availability of therapy. Highly fractionated total skin electron beam (TSEB) therapy is not available in many areas and as a result is often never considered as a treatment option. Similarly, a patient may live too far from a phototherapy facility to make that option practical. Photopheresis is also available only in major referral centers. One major feature that affects the selection of a goal is the probability of achieving that goal with a given treatment. With patch plaque disease, there are many options available for achieving remission in the majority of patients, whereas with erythroderma remission, it is only achieved in a minority of patients. Comorbidities also impact the selection of a therapy goal and course. Disabled patients have difficulty carrying out therapies that require prolonged standing, such as phototherapy of TSEB. Comorbid bone marrow diseases often preclude the use of cytotoxic and bone marrow-suppressing agents. Photosensitivity and ingestion of photoactive medications (like amiodarone) also can dampen the enthusiasm for phototherapy. Age also plays a role in goal and therapy selection. A patient with disseminated patch plaque disease at age 90 would more likely be treated with palliative therapy, whereas the same lesions in a 20-year-old patient would undergo aggressive phototherapy. Typically, the discussion of the goal of therapy incorporates these factors along with the patient's family. It is important to stress that goals can change. Often, the disease may prove resistant to remission, prompting a change to palliative therapy, or perhaps the marked improvement with a palliative therapy has generated interest in pursuing remission-plans change.

\section{Managing the Management Menus}

In preparing a patient's management plan, the first step is selecting a goal as discussed earlier in the text. The next step is to incorporate several time-honored principles of managing CTCL. The first is that localized disease tends to be managed with localized therapy, whereas widespread disease is met with either total skin therapy or systemic therapy. CTCL continues to weaken the T-cell immunity of the patient as long as it is active. ${ }^{3}$ Hence, immunosuppressive and cyto- toxic therapies tend to have greater complications and morbidity, and these agents are used more as backup therapies. And because CTCL patients have an illness that can span decades, there is a concern for treatments that have cumulative toxicities on keratinocytes (skin cancer) or the bone marrow (cytopenias). Each management menu presented will incorporate the majority of commonly used treatments. Those treatments that achieve the given response in the majority of patients are presented. Thus, even though remissions have been reported to occur occasionally with many of the palliative therapies, the inclusion of a therapy in the remittive category is based on its ability to induce that response in a majority of patients. After each menu, the therapies will be discussed in-depth in terms of administration, monitoring, and evaluating. Therapies mentioned in previous sections will be noted in the menus, but not discussed.

\section{Remission of Limited Disease}

- Radiation therapy

- Bexarotene gel

- Imiquimod cream

- Excimer laser

- Photodynamic therapy

- 5-Fluorouracil cream

Radiation therapy. Localized radiation therapy refers to superficial and orthovoltage x-rays given in doses between 20 and 36 Gy at individual fractions of 1-2 Gy per session. Isolated lesions can be cleared at success rates greater than any other therapy discussed in this review. The treatment of patients with limited disease with radiotherapy has led to disease-free survival intervals of $>8$ years, which is synonymous with cure. . $^{4,5}$

Bexarotene gel. Bexarotene gel is a retinoid that has a doselimiting toxicity of irritant dermatitis. Hence, the applications to a given lesion may vary from 1-3 applications per day. The erythematous response to the bexarotene gel is usually noticeable but not symptomatic. The dose-limiting irritant dermatitis also limits the amount of skin that can be treated. Typically, patients with 5\% body surface area involvement can tolerate the irritant effects. If the patient has discomfort, then the frequency should be reduced. Bexarotene gel therapy highlights many of the features of CTCL therapy. A patient is willing to undergo 12 weeks of an irritant dermatitis to clear the lesion, and unlike radiotherapy, the treatment may be conducted at home. As a retinoid, there is no synergy with other mutagenic treatments that tend to increase the risk of squamous cell carcinoma. The drug is not absorbed to any significant levels, but as a retinoid, it should not be used in pregnant patients. Patients will typically initiate therapy with nightly applications of bexarotene gel only to the lesions. After a week, the frequency is increased to twice daily and then thrice daily after another week. The irritant response makes monitoring the lesion(s) under therapy impossible. As a result, the response is typically maintained for 12 weeks and after a 4-week break, the therapeutic response can be assessed as complete clearing, partial re- 
sponse, or no response. Partial responses may warrant a second course of more intense therapy because the therapeutic effects appear to be dose responsive. ${ }^{6}$

Imiquimod cream. Imiquimod is a toll-like receptor agonist that locally stimulates the release of interferon (IFN). The 5\% cream is applied topically to the involved patches or plaques of disease. Although some have used daily application, the main side effect is a possible brisk inflammatory response with blister and pruritus. Because it is not possible to predict the severity of this reaction, initial therapy should be 3 days per week. Even at this frequency, some will develop significant skin inflammation and require decreased frequency. The frequency is increased as tolerated to daily dosing. In the case of severe inflammation, the treatment can be held for several days and then restarted. Rarely, the systemic absorption is sufficient to trigger symptoms of fatigue, fevers, or gastrointestinal symptoms. Response should be assessed after 12 weeks. Some will have a complete response in this time, whereas others with a partial response will need additional therapy. ${ }^{7,8}$ If there is no response after 12 weeks, alternative therapy should be considered.

Excimer laser. Excimer laser therapy for localized disease has been reported in the literature and is a logical choice, as the xenon-chloride laser produces ultraviolet light at 308 $\mathrm{nm}$, which is within the UVB spectrum. Indeed, the excimer has the benefit over standard phototherapy of providing localized therapy to minimize phototoxicity and allowing higher dosages because of the limited treatment areas. There are currently no defined protocols for the use of the excimer laser in MF. Ideally, a minimal erythema dose (MED) is obtained, and the starting dose is approximately the MED to twice the MED. When MED is not available, the currently available protocols for the treatment of plaque psoriasis with excimer laser can be used. Until a complete response occurs, treatment should be given twice weekly (BIW). This can be expected in $<12$ weeks in most patients who show response. ${ }^{9}$

Photodynamic therapy. Photodynamic therapy involves the use of topical photosensitizers with ultraviolet light. There have been more than 10 case reports and series showing that aminolevulinic acid combined with a light source is effective for limited MF. The treatment has been applied to patches and plaques; however, penetration of the topical preparation is best with thinner lesions that are $<7.5 \mathrm{~cm}$ in diameter. There has been no consistent protocol for treatment, although most reports have applied the topical agent for 3-6 hours. Light sources are also varied and include noncoherent light sources (eg, Waldmann PDT 1200), blue light systems (eg, Blu-U), pulsed dye laser, Nd-YAG laser, and others. Noncoherent/blue light systems are easier to administer because of the increased size of the treated area compared with laser light. ${ }^{10,11}$

5-Fluorouracil cream. 5-Fluorouracil (5-FU) is an analog of uracil that preferentially disrupts RNA and DNA synthesis in malignant cells. Topical 5-FU cream has been reported in a case series of 6 patients with CTCL who responded to therapy. Four patients had a complete response. The efficacy of topical 5-FU remains unknown; however, it is safe with few side effects. Some patients respond within 3 months, and therefore reassessment after 12 weeks of treatment is recommended. ${ }^{12}$

\section{Palliation of Limited Disease}

- Topical corticosteroids

- Wait and watch

Topical corticosteroids. The lympholytic and antiinflammatory properties of topical steroids make these a common adjunct to skin-directed therapy of CTCL. Clobetasol cream had its palliative properties described in a study of twicedaily applications for 2 months. ${ }^{13}$ Short-duration complete responses were common. The long-term follow-up of large numbers of patients is not yet available for this modality; hence, it cannot be compared with traditional treatments. However, given that there is an impact of topical steroids on the disease, these agents are commonly used as adjuncts to other treatments. The current role for topical steroids is usually as a palliative adjunct with triamcinolone for large areas of body surface involvement and clobetasol for limited areas. Another crucial role played by the class I steroids is in the aggressive treatment of any suspicious early relapse. Patients are instructed to treat any eruption they may encounter (contact dermatitis and insect bites) for a maximum of 2 months. Should lesions persist, then therapy is stopped and a biopsy performed.

Wait and watch. With other noncutaneous indolent lymphomas, the approach of "wait and watch" is common and an accepted approach to managing a patient. In the setting of limited CTCL, this translates into waiting until the disease becomes more widespread or tumorous to warrant intervention. The approach of wait and watch tends to be used more in older patients where other events may transpire before the CTCL would impact the patient's well-being.

\section{Remission of Widespread patch-plaque MF (T2)}

- Phototherapy

- Nitrogen mustard (NM)

- TSEB

- Allogeneic stem cell transplant

Widespread disease reflects a need for total skin therapy, including the treatment of uninvolved skin. The therapies that can induce remissions in most patients treated are discussed here.

Phototherapy. Phototherapy has probably been used more than any other modality to achieve remissions of MF. However, the type of phototherapy used has been changing because of the lack of availability of the best studied and most commonly reported form of photochemotherapy with oxsoralen and ultraviolet A light (PUVA). ${ }^{14,15}$ With the psoriasis market turning more to injectable biologics, PUVA has lost 
market share and presence. In its place, narrowband ultraviolet B phototherapy (NBUVB) has grown to be the most widely available form of phototherapy, with results that are similar to PUVA. ${ }^{16}$ The principles of using PUVA, NBUVB, and broadband ultraviolet $B$ are the same, although efficacy is generally in that order of presentation.

For inducing a remission, treatments should begin at 3 times per week frequency. It is important to observe some degree of phototoxicity to ensure the patient is receiving a dose of light that will be lympholytic. In the course of PUVA treatment, there may be poor absorption of the oral photosensitizer, and the oral psoralen dose may need to be increased. After most of the lesions have cleared, the frequency can be decreased to BIW until the patient has achieved a complete remission. It is important to maintain the multiple treatments per week schedule for a minimum of 3 and a maximum of 6 months. Another form of NBUVB that is increasing in use is home phototherapy with NBUVB. ${ }^{17}$ This requires an extremely well-informed and diligent patient. Typically, home-based treatments can be done 7 days per week to maximize the dose-responsiveness of phototherapy.

With a partial phototherapy response, there are several options to boost the therapeutic efficacy. Two of the more traditional choices at this point are retinoid therapy and IFN therapy. Both are proven in the role of enhancing a response to phototherapy. IFN alpha doses ranging from 3 to $18 \mathrm{~m} \mathrm{U}$ subcutaneously 3 times per week can act synergistically with PUVA clear refractory disease. ${ }^{18}$ The side effects of IFN are discussed later. For those who cannot tolerate IFN or for those who prefer an alternative, retinoid-PUVA therapy can also be successful. Bexarotene (discussed later) at dosages of $150-450 \mathrm{mg} / \mathrm{d}$ has been used in conjunction with phototherapy to achieve remission. ${ }^{19}$ Retinoids increase the risk of phototoxicity, and ultraviolet light doses are typically reduced during the induction of bexarotene therapy. With retinoid and/or IFN phototherapy boosters, the light treatments can usually maintain the remission after the adjunct is stopped.

As a maintenance therapy, phototherapy is initially administered at once-weekly frequencies until 1 year has passed. At this point, 1 week can be added between treatments, and a total of 25 sessions over a year will be given over the second year of maintenance. Should the patient still be in remission at this point, the same addition is carried out over 2 more years, so that eventually patients receive 12 sessions per year for at least 2 years. At this point, the patient should have been in remission for 5 years. Once a patient has achieved complete control with 12 sessions per year, the continued use of maintenance is balanced against evidence of phototoxicity, photocarcinogenesis, and the possibility that a relapse may necessitate a return to multiple sessions per week therapy.

With all the phototherapies, the most easily exposed lesions clear rapidly (patches on the middle of the trunk), but all phototherapies may have sanctuaries. Lesions that may persist deep in the groin, on the ankles, buttock crease, and the eyelids do not represent therapy failure as much as they represent a sanctuary from the skin-directed therapy. These often require adjunctive therapy to clear, typically with localized therapies discussed in the menu for limited disease.

Nitrogen mustard. Topical NM, mechlorethamine hydrochloride, is an alkylating agent that is also considered a radiomimetic in that its lympholytic properties mimic ionizing radiation. And the mechanism by which it exerts its therapeutic effect on the skin is not well understood. Many of the initial studies used the aqueous solution of NM that was prepared by the patient daily for whole-body application. ${ }^{20}$ With the current shortage of NM, this approach has been supplanted by the use of an ointment formulation. It is compounded by specialty pharmacies at $10 \mathrm{mg} / \%$ in petrolatum and is stable at room temperature for long periods. ${ }^{21}$ The patient applies the NM ointment daily to the entire body. By being one of the first regimens of skin-directed therapy for CTCL, there is excellent long-term follow-up of NM-treated patients. Remission is reliable ( $>50 \%$ ), but the current consensus is that long-term maintenance regimens are needed to suppress recurrence; however, there is no consensus on tapering regimen to maintenance therapy.

Other than the discomfort of whole-body ointment application, the side effects from NM therapy include primary irritant reactions, development of second cutaneous malignancies, and hypo- and hyperpigmentation, especially in intertriginous areas. Cutaneous malignancies in sun-protected areas of patients treated with NM have also been documented. To minimize carcinogenic effects of NM, it is not used with phototherapy, and patients are advised to limit sun exposure while using NM. The most recent advance in NM therapy is the use of BIW applications in conjunction with topical steroid therapy. This regimen minimizes the discomfort of nightly applications and achieved a remission in most patients treated.22

TSEB. Radiotherapy for disseminated disease needs to incorporate treatment of clinically normal skin, much like phototherapy and NM. Since electrons penetrate only to the upper dermis, electron radiation in the form of TSEB therapy may be used without systemic effect. The total dose of irradiation is important, and a dose of $30 \mathrm{~Gy}$ or more gives better complete remission rates and disease-free survival than lower doses. With a 6-field technique, it is possible to administer a course of therapy for 9 weeks. Those areas not well exposed with the 6-field approach need additional therapy: soles, scalp, perineum, and flexural areas in obese patients. The major disadvantages are that this type of therapy requires a specialized center, and it takes up to 3 months for complete treatment in the case of TSEBT. Local side effects may include alopecia, atrophy of sweat glands and skin, radiodermatitis, and edema. When the total dose is highly fractionated, these complications are minimized and sometimes avoided. ${ }^{23}$ The question then arises as to what is the maximum radiation tolerance of the skin. Using the highly fractionated approach, patients can receive a second course of 36-Gy electron beam to reinduce a remission. ${ }^{24}$ As the total radiation dose increases, so does the risk of squamous cell carcinoma and radiodermatitis. 
TSEB brings about complete remission at rates higher than any other skin-directed therapy. As with other modalities, the response is stage dependent, with limited disease having higher response rates than disseminated disease. Most of the relapses occur within the first year after completion of therapy and are relatively rare after 3 years. Thus, phototherapy maintenance is often instituted to prevent relapse.

Allogeneic stem cell transplant. Reduced-intensity allogeneic stem cell transplant, preferably from a matched sibling, takes advantage of the graft versus lymphoma aspects of graft-versus-host disease to control the CTCL. With reduced intensity preparation, there is often residual disease in the skin at the time of transplantation. ${ }^{25}$ In the peritransplant period, patients are exposed to systemic immunosuppressants to help control the graft-versus-host disease. The immunosuppression to treat graft-versus-host disease can accelerate the CTCL so that most patients will need adjunctive therapy that does not compromise the engrafting bone marrow. Oral bexarotene, phototherapy, and radiation therapy have all been used to get patients through the peritransplant period. In the months following engraftment, a gradual reduction of disease burden occurs, and typically a complete remission is achieved. ${ }^{26,27}$

\section{Palliation of Widespread Patch Plaque MF (T2)}

Menu for palliation of widespread patch-plaque MF (T2)

- Oral methotrexate

- Oral bexarotene

- Oral vorinostat

- Romidepsin

- Denileukin diftitox

- Liposomal doxorubicin

Palliative therapy for MF is measured by the impact of the therapy and the disease on the quality of life. Thus, most therapies used for palliation do not interrupt daily activities (like phototherapy), cause hair loss (like TSEB), or cause depression (like IFN). Oral treatments can be administered at home, and intermittent monitoring of laboratory parameters is the only interruption. There are 3 items on this menu that require infusion, but even those are well tolerated and easily monitored.

Oral methotrexate. Once weekly, methotrexate has been successfully used for MF in doses from 5 to $35 \mathrm{mg}$ orally or subcutaneously. Therapeutic responses should be assessed by quality of life parameters within 3 months of starting therapy. ${ }^{28}$ Weekly methotrexate can be sustained as long as the therapy is tolerated. The dosing and toxicity of weekly methotrexate are similar to that with psoriasis. Hematologic and liver parameters are important to follow for evidence of antifolate toxicity. The renal parameters need to be followed because this is the primary route of excretion for the drug. Monitoring for hepatitis or leukopenia is recommended at the same intervals used for psoriasis patients on methotrex- ate. If no abnormalities occur, less frequent monitoring of at least once every 3 months is recommended thereafter.

Oral bexarotene. Bexarotene is an oral retinoid typically used as monotherapy. There are 2 side effects that must be addressed in all patients. All patients will have suppression of thyrotropin-stimulating hormone, and this requires levothyroxine replacement therapy and monitoring of free T4 levels. The second is the dose-limiting side effect of hypertriglyceridemia and hypercholesterolemia.

If not adequately controlled, hypertriglyceridemia can cause pancreatitis, which, like the other side effects, is reversible with the discontinuation of bexarotene. In addition, oral bexarotene, like other oral retinoids, is contraindicated in women who are pregnant or planning a pregnancy.

The benefits of bexarotene therapy on the quality of life have been well documented in clinical trials..$^{29,30}$ The overall strategy is to continue bexarotene as long as the desired improvement in quality of life is achieved. Bexarotene reliably is palliative and rarely remittive as a monotherapy. In combination with IFN and/or phototherapy, it can be a component of a remittive regimen. ${ }^{19}$ Another advantage of combining bexarotene with other therapies is that lower doses of the retinoid can be used, and the lipid abnormalities are less of a problem.

Oral vorinostat. Vorinostat is the first of a class of histone deacetylase inhibitors to be used in malignant diseases. This class of drugs is nonimmunosuppressing; the clinical effects are those of a palliative agent; and it can reduce pruritus and improve quality of life. Vorinostat is an oral agent that was studied in patients seeking palliation who had failed to respond to or been intolerant of oral bexarotene. Monotherapy trials have shown the safest and most effective oral dose is $400 \mathrm{mg} / \mathrm{d}$. Although the demonstrable benefits occur in slightly less than the majority of patients, most of the patients managed with oral vorinostat have been pretreated with other agents that have failed and thus have fairly resistant disease. The side effect profile is acceptable as a palliative agent. Thrombocytopenia can occur and necessitates repeated blood monitoring. Dehydration and diarrhea are 2 more common side effects that can be screened for by symptoms. ${ }^{31}$ There have been no synergy studies with vorinostat. The current usage is as a palliative agent when remissions cannot be safely achieved in symptomatic patients. Vorinostat can be used for long-term management unless either the side effects or the disease becomes unmanageable. ${ }^{32}$

Romidepsin. The second histone deacetylase inhibitor to be approved for palliation of CTCL is romidepsin, an infusional form of this class of drugs. Romidepsin is usually given at doses of $14 \mathrm{mg} / \mathrm{M} 2$ once a week for 3 weeks to complete a 4 -week cycle. These monthly cycles can be repeated, and usually 2 are needed to determine whether the patient is having a sufficient response. Patients will generally experience electrocardiographic changes, such as QT interval prolongation. As a result, pre- and postcardiograms are routine along with eliminating any reversible causes of QT-segment prolongation. Like vorinostat, there is a generalized sense of 
fatigue that patients often experience. Bone marrow suppression, dehydration, and thrombotic phenomena appear to be the toxicity profile of this class, and appropriate monitoring is advised. The most reliable response is with pruritus, with almost half the patients reporting improvement. ${ }^{33}$

Denileukin difititox. Denileukin diftitox was the first infusional therapy approved for palliation of CTCL. It is a recombinant protein produced from fusing the gene for diphtheria toxin with the gene for interleukin 2. Denileukin diftitox is given as an intravenous infusion for 5 consecutive days ( 9 or $18 \mu \mathrm{g} / \mathrm{kg} / \mathrm{d}$ ) and then repeated after 21 days off drug. ${ }^{34}$ After the third cycle of therapy, responders will exhibit an improvement in the quality of life, and the cycles continued. ${ }^{35}$ It is important to stress the need for peripheral vein access as opposed to indwelling lines for drug administration. Patients with CTCL tend to develop sepsis and infected catheters following implantation of long-term indwelling catheters. The most frequent severe toxicity of denileukin diftitox was edema from the vascular leak syndrome. Cachectic patients with low albumin levels are at increased risk for this side effect. Currently, use of steroid premedication has significantly reduced the frequency of this complication. ${ }^{36}$ Another common toxicity is a reversible elevation of liver enzymes that reaches a peak during the week following an infusion. This will usually return to normal by the start of the second infusion, and any subsequent elevations are typically of lower magnitude. Otherwise, significant flu-like symptoms or acute hypersensitivity reactions are much less frequently seen in the setting of steroid premedication. Responding patients continue to repeat cycles of therapy until a maximal response has been achieved.

Liposomal doxorubicin. Liposomal doxorubicin is pegylated liposomal encapsulated formulation of doxorubicin, which has been shown to reduce toxicity as compared with standard doxorubicin therapy. The medication is delivered by intravenous infusion and doses of $20 \mathrm{mg} / \mathrm{m}^{2}$ every 4 weeks. Palliative responses occurred in the majority of patients. This compares favorably against reported response rates for multiagent regimens incorporating anthracycline derivatives that have more immunosuppressive side effects. Liposomal doxorubicin monotherapy was tolerated very well, with severe toxicity stemming only from marrow suppression toxic erythema in $12 \%$ of patients. Total cumulative dose, less than $450-550 \mathrm{mg} / \mathrm{m}^{2}$, is recommended to avoid doxorubicin-induced cardiomyopathy. ${ }^{37,38}$

\section{Remission of Erythrodermic MF/Sezary Syndrome (T4) \\ - Stem cell transplant}

\section{Palliation of Erythrodermic MF/Sezary Syndrome}

- Oral bexarotene

- Oral methotrexate

- Oral vorinostat

- Romidepsin
- Photopheresis

- IFN

Erythrodermic forms of CTCL often have devastating impacts on quality of life. Unrelenting pruritus, obvious skin lesions, uncontrolled temperature balance along with palm and sole involvement conspire to make life miserable. Another depressing feature is that durable long-term remissions are only reliably achieved with stem cell transplantation and its attendant risks. Erythrodermic disease is usually associated with demonstrable immunosuppression. Patients with this form of the disease are markedly susceptible to infections (central lines are contraindicated) and to the side effects of any immunosuppressive side effects of treatments. The management of the erythrodermic patient begins with the assessment of whether there is an acceptable risk of successfully transplanting stem cells from an appropriately matched donor (preferably sibling). Most patients are then considered for palliative therapy. In addition to the nonimmunosuppressing bexarotene, vorinostat, and romidepsin therapies, there is another modality used preferentially in erythrodermic patients known as photopheresis.

Photopheresis. Photopheresis therapy involves the photoinactivation of a portion of a patient's lymphocyte compartment followed by reinfusion of these cells. As with most therapies that have undergone clinical trials to establish efficacy, the regimens for using photopheresis reflect the schedules used in the initial multicenter clinical trials. Patients are treated on consecutive half days for 2 days, and then 4 weeks later, the cycle is repeated. This treatment is available at select hospitals and cancer centers, making availability a problem that compromises its widespread long-term use.

Erythrodermic patients beginning on photopheresis should be initially reevaluated after 3 cycles of therapy. By this point, a trend in their clinical status should be evident. If there are signs of improvement, the therapy should be continued to maximize the therapeutic response. ${ }^{39}$ Patients having incomplete responses at 3-6 months should be considered for adjunctive therapy if the level of palliation is not sufficient. Oral bexarotene and injectable IFN are the 2 most common adjuncts, and the use of all 3 immunomodulatory therapies has been shown to achieve impressive responses. ${ }^{40}$

IFN. IFN alpha has shown activity against all stages of MF, but remissions do not occur in a majority of patients. The dose, frequency, maintenance period, and tapering regimen of IFN alpha varied from 1 study to another, and thus no standardized regimen is universally accepted. Generally, doses of 3 million $U 3$ times a week are used, and the response to that judged at 3-month intervals. Once there is a maximal response, the dose is tapered over 12 months either by frequency of administration (ie, thrice weekly (TIW) to BIW) or by dose to minimize side effects. ${ }^{41}$ IFN alpha has both acute and chronic side effects. Initially and almost invariably, the patient will experience flu-like symptoms, including malaise, myalgia, headache, fever, and listlessness. Warning the patient of these symptoms as well as bedtime dosing and acetaminophen pretreatment could reduce this 
acute toxicity. Autoimmune phenomena affecting the blood counts, renal status, thyroid function, and neurologic status are monitored routinely. For the most part, the side effects are reversible and require dose adjustment.

\section{Remission of Tumors of MF (T3)}

- Stem cell transplant

- Radiotherapy: localized and TSEB

\section{Palliation of Tumors}

Although tumors are readily recognized by the clinician, there is no accepted definition. Typically, the appearance of a tumor signifies the disease has acquired a significant vertical growth phase.

- Oral bexarotene

- Oral methotrexate

- Oral vorinostat

- Denileukin diftitox

- Romidepsin

- Liposomal doxorubicin

\section{Treatment of Non-MF CTCLs and Peripheral T-cell Lymphomas}

- Radiotherapy

- Stem cell transplant

- Denileukin diftitox

- Liposomal doxorubicin

- Pralatrexate

Pralatrexate. The CTCLs that are categorized as non-MF CTCLs and peripheral T-cell lymphomas are defined by their histologic properties. This category includes pleomorphic CTCL, anaplastic large cell lymphoma, panniculitic lymphomas, NK lymphomas, and unclassifiable variants. Therapy is typically with infusional treatments with or without radiotherapy, and consideration is always given to the risks and benefits of allogeneic stem cell transplantation. These entities are rare, and it is not known whether disease reduction can improve survival or quality of life. Hence, the treatments for these CTCLs are lumped together, as there is no clear information on palliation or survival. If there is limited disease, radiation is typically used to see whether a durable remission can be induced. If the response is not durable or the disease is widespread, then infusional treatments are used. A newly approved antifolate, pralatrexate, has been approved to treat peripheral T-cell lymphomas.

Antifolates have been useful in the treatment of lymphoid malignancies since the initial discovery of aminopterin as a therapy for lymphocytic leukemia. The second-generation antifolate methotrexate has already been discussed as a useful palliative agent for MF and Sezary syndrome. Pralatrexate has a high affinity for one of the folate transport proteins and is internalized more than previously available antifolates. The toxicities can be ameliorated with adjunctive oral folates and B-12 injections. Pralatrexate is given in cycles of weekly infusions (typically, $30 \mathrm{mg} / \mathrm{M} 2$ ) done once weekly for 6 weeks. At the end of a cycle of therapy, the tumor burden can be reassessed to determine the response. Mucositis, leukopenia, and thrombocytopenia are the limiting side effects. Cycles of therapy can be repeated to maximize the response. ${ }^{42}$

\section{Conclusions}

During the past 20 years, 5 therapies have been approved for the management of CTCL. With the exception of the bexarotene gel, these new therapies have been primarily palliative and intended for more advanced disease. Remissions and cures are routine with traditional therapies in early disease. Although the field may appear to be cluttered with options, what is not cluttered is the goal of therapy. Agents have been described by their remittive or palliative properties, allowing physicians to use an appropriate therapy for a given goal. The next developments will be with the emergence of combination therapies for the more difficult to remit versions of CTCL. The value of a combination therapy can be assessed by minimizing risk, maximizing response, or reflected in the patient's preference by way of assessing the impact of therapy and disease on the quality of life.

\section{References}

1. Girardi M, Heald PW, Wilson LD: The pathogenesis of mycosis fungoides. N Engl J Med 350:1978-1988, 2004

2. Heald PW: Memorials and mandates for cutaneous lymphomas. Arch Dermatol 139:926-928, 2003

3. Rook A, Heald P: The immunopathogenesis of cutaneous T-cell lymphoma. Hematol Oncol Clin North Am 9:997-1010, 1995

4. Wilson LD, Kacinski BM, Jones GW: Local superficial radiotherapy in the management of minimal stage IA cutaneous T-cell lymphoma (mycosis fungoides). Int J Radiat Oncol Biol Phys 40:109-115, 1998

5. Heald PW, Glusac EJ: Unilesional cutaneous T-cell lymphoma: Clinical features, therapy, and follow-up of 10 patients with a treatment-responsive mycosis fungoides variant. J Am Acad Dermatol 42:283-285, 2000

6. Heald P, Mehlmauer M, Martin AG, et al: Topical bexarotene therapy for patients with refractory or persistent early-stage cutaneous T-cell lymphoma: Results of the phase III clinical trial. J Am Acad Dermatol 49:801-815, 2003

7. Suchin KR, Junkins-Hopkins JM, Rook AH: Treatment of stage IA cutaneous T-cell lymphoma with topical application of the immune response modifier imiquimod. Arch Dermatol 138:1137-1139, 2002

8. Deeths MJ, Chapman JT, Dellavalle RP, et al: Treatment of patch and plaque stage mycosis fungoides with imiquimod 5\% cream. J Am Acad Dermatol 52:275-280, 2005

9. Nisticò S, Costanzo A, Saraceno R: Efficacy of monochromatic excimer laser radiation $(308 \mathrm{~nm})$ in the treatment of early stage mycosis fungoides. Br J Dermatol 151:877-879, 2004

10. Wolf P, Fink-Puches R, Cerroni L, et al: Photodynamic therapy for mycosis fungoides after topical photosensitization with 5-aminolevulinic acid. J Am Acad Dermatol 31:678-680, 1994

11. Edström DW, Hedblad MA: Long-term follow-up of photodynamic therapy for mycosis fungoides. Acta Derm Venereol 88:288-290, 2008

12. Kannangara AP, Levitan D, Fleischer AB Jr: Six patients with early-stage cutaneous T-cell lymphoma successfully treated with topical 5-fluorouracil. J Drugs Dermatol 9:1017-1018, 2010

13. Zackheim HS, Kashani-Sabet M, Amin S: Topical corticosteroids for mycosis fungoides. Experience in 79 patients. Arch Dermatol 134:949954, 1998

14. Swanbeck G, Roupe G, Sandstrom MH: Indication of a considerable decrease in the death rate in mycosis fungoides by PUVA therapy. Acta Derm Venereol 74:465-466, 1994

15. Roupe G, Sandstrom MH, Kjellstrom C: PUVA in early mycosis fungoi- 
des may give long-term remission and delay extracutaneous spread. Acta Derm Venereol 76:475-478, 1996

16. Ponte P, Serrão V, Apetato M: Efficacy of narrowband UVB vs. PUVA in patients with early-stage mycosis fungoides. J Eur Acad Dermatol Venereol 24:716-721, 2010

17. Resnik KS, Vonderheid EC: Home UV phototherapy of early mycosis fungoides: Long-term follow-up observations in thirty-one patients. J Am Acad Dermatol 29:73-77, 1993

18. Nikolaou V, Siakantaris MP, Vassilakopoulos TP, et al: PUVA plus interferon Alpha2B in the treatment of advanced or refractory to PUVA early stage mycosis fungoides: A case series. J Eur Acad Dermatol Venereol 25:354-357, 2011

19. Singh F, Lebwohl MF: Cutaneous T-cell lymphoma treatment using bexarotene and PUVA: A case series. J Am Acad Dermatol 51:570-573, 2004

20. Vonderheid EC, Tan ET, Kantor AF, et al: Long-term efficacy, curative potential, and carcinogenicity of topical mechlorethamine chemotherapy and cutaneous T-cell lymphoma. J Am Acad Dermatol 20:416-428, 1989

21. Price NM, Hoppe RT, Deneau DG: Ointment based mechlorethamine treatment for mycosis fungoides. Cancer 52:2214-2219, 1983

22. de Quatrebarbes J, Estève E, Bagot M, et al: Treatment of early-stage mycosis fungoides with twice-weekly applications of mechlorethamine and topical corticosteroids: A prospective study. Arch Dermatol 141: $1117-1120,2005$

23. Jones G, Wilson LD, Fox-Goguen L: Total skin electron beam radiotherapy for patients who have mycosis fungoides. Hemtol Oncol Clin 17:1421-1434, 2003

24. Wilson LD, Quiros PA, Kolenik SA, et al: Additional courses of total skin electron beam therapy in the treatment of patients with recurrent cutaneous T-cell lymphoma. J Am Acad Dermatol 35:69-73, 1996

25. Herbert KE, Spencer A, Grigg A, et al: Graft versus lymphoma effect in refractory cutaneous $\mathrm{T}$ cell lymphoma after reduced intensity HLA matched sibling allogeneic stem cell transplantation. Bone Marrow Translplant 34:521-525, 2004

26. Duarte RF, Canals C, Onida F, et al: Allogeneic hematopoietic cell transplantation for patients with mycosis fungoides and Sezary syndrome: A retrospective analysis of the lymphoma working party of the European group for blood and marrow transplantation. J Clin Oncol 28:4492-4499, 2010

27. Molina A, Zain J, Arber DA, et al: Durable clinical, cytogenetic, and molecular remissions after allogeneic hematopoietic cell transplantation for refractory Sezary syndrome and mycosis fungoides. J Clin Oncol 23:6163-6171, 2005

28. Zackheim HS, Kashani-Sabet M, McMillan A: Low-dose methotrexate to treat mycosis fungoides: A retrospective study in 69 patients. J Am Acad Dermatol 49:873-878, 2003
29. Duvic M, Hymes K, Heald P, et al: Bexarotene is effective and safe for treatment of refractory advanced-stage cutaneous T-cell lymphoma: Multinational phase II-III trial results. J Clin Oncol 19:2456-2471, 2001

30. Duvic M, Martin AG, Kim Y, et al: Phase 2 and 3 clinical trial of oral bexarotene (Targretin capsules) for the treatment of refractory or persistent early-stage cutaneous T-cell lymphoma. Arch Dermatol 137: 581-593, 2001

31. Duvic M, Talpur R, Ni X, et al: Phase 2 trial of oral vorinostat (suberoylanilide hydroxamic acid, SAHA) for refractory cutaneous T-cell lymphoma (CTCL). Blood 109:31-39, 2007

32. Duvic M, Olsen EA, Breneman D, et al: Evaluation of the long-term tolerability and clinical benefit of vorinostat in patients with advanced cutaneous T-cell lymphoma. Clin Lymphoma Myeloma 9:412-416, 2009

33. Piekarz RL, Frye R, Turner M, et al: Phase II multi-institutional trial of the histone deacetylase inhibitor romidepsin as monotherapy for patients with cutaneous T-cell lymphoma. J Clin Oncol 27:5410-5417, 2009

34. Olsen E, Duvic M, Frankel A, et al: Pivotal phase III trial of two dose levels of denileukin diftitox for the treatment of cutaneous T-cell lymphoma. J Clin Oncol 19:376-388, 2001

35. Duvic M, Kuzel TM, Olsen EA, et al: Quality of life improvements in cutaneous T cell lymphoma patients treated with denileukin diftitox (ONTAK). Clin Lymphoma 2:222-228, 2002

36. Foss FM, Bacha P, Osann KE, et al: Biological correlates of acute hypersensitivity events with DAB(389)IL-2 (denileukin diftitox, ONTAK) in cutaneous T-cell lymphoma: decreased frequency and severity with steroid premedication. Clin Lymphoma 1:298-302, 2001

37. Lybaek D, Iversen L: Pegylated liposomal doxorubicin in the treatment of mycosis fungoides. Acta Derm Venereol 86:545-547, 2006

38. Wollina U, Dummer R, Brockmeyer NH, et al: Multicenter study of pegylated liposomal doxorubicin in patients with cutaneous T-cell lymphoma. Cancer 98:993-1001, 2003

39. Heald P, Rook A, Perez M, et al: Treatment of erythrodermic cutaneous T-cell lymphoma patients with extracorporeal photochemotherapy. J Am Acad Dermatol 27:427-433, 1992

40. Richardson SK, Lin JH, Vittorio CC, et al: High clinical response rate with multimodality immunomodulatory therapy for Sézary syndrome. Clin Lymphoma Myeloma 7:226-232, 2006

41. Dreno B, Godefroy WY, Fleischmann M, et al: Low-dose recombinant interferon-alpha in the treatment of cutaneous T-cell lymphoma. Br J Dermatol 121:543-544, 1989

42. O'Connor OA, Pro B, Pinter-Brown L, et al: Pralatrexate in patients with relapsed or refractory peripheral T-cell lymphoma: Results from the pivotal PROPEL study. J Clin Oncol 29:1182-1189, 2011 